\title{
Contribuciones de Zamorano a la Educación y Preparación de Personal Técnico en América
}

\author{
Carla Henríquez ${ }^{1}$, Rosa Amada Zelaya ${ }^{2}$ y Hugo Zavala ${ }^{3}$
}

Resumen. Zamorano fue fundada hace 70 años con la visión de formar profesionales en la rama agrícola. Sus acciones de formación están enfocadas a mantener la calidad de sus programas para responder a las particularidades de un entorno actual y cambiante. A través de su historia, y bajo el esquema de un plan académico riguroso y disciplinario, la filosofía de enseñanza zamorana combina el aprendizaje en el salón de clases y la práctica de campo (Aprender-haciendo) con la formación en valores, que caracterizan a los graduados zamoranos. Los docentes de Zamorano son contribuyentes activos de esta formación panamericana centrada en valores. La investigación técnica desarrollada por Zamorano y la proyección de esa investigación en actividades tangibles, ha logrado que Zamorano genere impactos positivos en muchas comunidades. De estas acciones, existen muchos ejemplos que han contribuido en el combate a la reducción de la pobreza y al detenimiento de la degradación ambiental. Los graduados de Zamorano han encontrado su espacio en la estructura productiva de los países que los reciben y contribuyen al logro de los planes de sus organizaciones y de los gobiernos. Todo esto y más definen el sello Zamorano.

Palabras clave: Aprender-haciendo, curriculum, Escuela Agrícola Panamericana, graduados, historia, investigación, proyectos.

\section{Zamorano Contributions to Education and Preparation of Technical Staff in America}

\begin{abstract}
Zamorano was founded 70 years ago with a vision to train professionals in agriculture. Training activities are focused on maintaining the quality of its programs to respond to the particularities of current and changing context. Through its history and under the scheme of a rigorous academic and disciplinary teaching philosophy, Zamorano combines learning in the classroom and practical experience in the field (learning by doing) with the formation of values, which characterize the Zamorano graduates. Zamorano teachers are active contributors to this Pan-American training focused on values. The research activities developed by Zamorano staff members and the projection of that research into tangible activities has managed to generate positive impacts in many communities. Of those activities, there are plenty of examples that have helped in the fight against poverty and the reduction of environmental degradation. Zamorano graduates have found their place in the productive structure of the countries in ehich they work contributing to the achievement of the plans of their organizations and governments. All this and more define the Zamorano seal.
\end{abstract}

Key words: Curriculum, Escuela Agrícola Panamericana, graduates, history, learning-by-doing, projects, research.

\section{Evolución de Zamorano}

Después de una larga trayectoria de 70 años desde su fundación, Zamorano ha evolucionado de una escuela práctica vocacional a una universidad de prestigio internacional, reconocida por su innovadora filosofía educativa estratégicamente combinada con un proceso de mejora continua que le permite ir de la mano con un mundo altamente cambiante, necesitado cada vez más de nuevos profesionales, investigadores y emprendedores con amplios conocimientos, muy disciplinados y con valores.

Para comprender este proceso evolutivo, es importante remontarnos a sus inicios y mencionar las contribuciones que ha logrado compartir con el mundo en la búsqueda de soluciones aplicables a los países que sirve.

En septiembre de 1943, la Escuela Agrícola Panamericana, mejor conocida como Zamorano, abrió sus puertas para un año de prueba y experimentación y

1 Jefe de Currículo y Desarrollo Docente Escuela Agrícola Panamericana, P.O. Box 93, Tegucigalpa. Honduras. chenriquez@zamorano.edu

2 Profesora Asociada, Escuela Agrícola Panamericana, P.O. Box 93, Tegucigalpa. Honduras. rzelaya@zamorano.edu

3 Jefe de Registro y Asistencia Financiera, Escuela Agrícola Panamericana, P.O. Box 93, Tegucigalpa. Honduras. hzavala@zamorano.edu

DOI: $10.5377 /$ ceiba.v52i1.971 
desde entonces, han estado abiertas para recibir estudiantes procedentes, principalmente, de países latinoamericanos, manteniendo la esencia y principios bajo los que fue creada.

Oficialmente, la construcción del primer edificio de Zamorano inició el 1 de marzo de 1942 a cargo del Ing. Harlo Van Wald y el Arq. Mario Valenzuela; tardó 14 meses hasta que fue concluido y es así como se sentaron las bases de la infraestructura para esta institución (Malo 1999: 195-198).

Desde su fundación, Zamorano, ha sufrido transformaciones y ajustes, administrativos y educativos, que le han permitido mantenerse como una de las mejores universidades agrícolas en Latinoamérica. Han sido 70 años de esfuerzos que redundan en un cuerpo estudiantil decidido y en graduados de muy alta calidad, ejemplo vivo del producto Zamorano. Es difícil hacer un detalle de todos los eventos administrativos, gerenciales, curriculares teóricos y prácticos, de proyección y de vida en el campus, han marcado la historia de Zamorano. El cuadro 1 presenta un resumen de los principales eventos y cambios en la historia de Zamorano.

\section{EI Sello Zamorano}

La institución, a través de los años, ha mantenido cuatro pilares que fundamentan todo lo que es la filosofía zamorana; desde su fundación, sus dos ávidos fundadores, Dr. Wilson Popenoe y Samuel Zemurray, establecieron que su metodología de enseñanza estaría fundamentada principalmente en el aspecto práctico (esa es la razón de la filosofía Aprender Haciendo soportado por la el lema Labor omnia vincit, el trabajo lo vence todo) que estaría al servicio de las Américas con una visión de formación integral y no sólo técnica.

Zamorano dedica mucho esfuerzo para asegurar que lo práctico esté en un proceso de mejora continua. El componente del Aprender Haciendo tiene como soporte 14 empresas universitarias y 24 unidades académicas (laboratorios y unidades de investigación) que son el escenario ideal para desarrollar en el estudiante el valor al trabajo, competencias técnicas y generales, y principalmente, formación de carácter y liderazgo. El Aprender Haciendo es manejado por los docentes que imparten las clases magistrales en las aulas que enfocan sus conocimientos técnicos en hacer práctica las labores con una base científica. Este es uno de los componentes que da un valor agregado a nuestros graduados, que sumado a la formación integral y liderazgo, es el sello que cada uno de ellos recibe y por el cual fácilmente logran diferenciarse y sobresalir. Es importante rescatar los esfuerzos que se han realizado en matizar este componente con las nuevas corrientes pedagógicas metodológicas convirtiéndolo en un modelo, ejemplo para especialistas en educación.

Zamorano ofrece un programa académico que tiene todo un fuerte componente teórico (lo que vendría a ser una universidad tradicional), pero al mismo tiempo, un componente completamente práctico (Aprender haciendo) independiente $\mathrm{e}$ interrelacionado que convierte a Zamorano en una institución única y diferente, y le da al estudiante 2 años de experiencia laboral real. El Aprender Haciendo tiene su propia planificación y ejecución y usa el $50 \%$ del tiempo de los estudiantes, además, apoya fuertemente el aprendizaje de las asignaturas que se desarrollan en las aulas de clases. No obstante, es un programa paralelo que posee su propia planificación académica y didáctica.

Entre las características especiales del cuerpo docente, pieza fundamental para mantener la excelencia académica, resalta el hecho que todos ellos están contratados a tiempo completo, lo que permite explotar el potencial científico y académico para beneficio del aprendizaje de los estudiantes. Estos docentes proceden de más de 18 países, lo que permite crear experiencias y vivencias mundiales que les abre a los estudiantes la curiosidad y visión global e integrada de la realidad a la que se enfrentarán contribuyendo a su diferenciación entre otros profesionales. Además, el cuerpo docente está muy calificado, poseen un alto nivel académico $(30 \%$ de los docentes tienen Ph.D.) y mucha experiencia en producción, investigación y desarrollo, lo que convierte el aula de clases y el aprender haciendo en escenarios prácticos y vivos que reta a los estudiantes y les da una experiencia profesional única. 
Cuadro 1. Resumen de la evolución de la Escuela Agrícola Panamericana, Zamorano, Honduras, de 1942 al 2011.

\begin{tabular}{|c|c|c|c|c|c|}
\hline Período & Resumen & $\begin{array}{c}\text { Currículum } \\
\text { Formal }\end{array}$ & $\begin{array}{l}\text { Aprender } \\
\text { Haciendo } \\
\end{array}$ & Proyección & $\begin{array}{c}\text { Ambiente del } \\
\text { Campus }\end{array}$ \\
\hline $\begin{array}{l}1942- \\
1957\end{array}$ & $\begin{array}{l}\text { Fundación de Zamorano. } \\
\text { Pautas principales: Labor } \\
\text { Omnia Vincit (el trabajo lo } \\
\text { vence todo), el aprender- } \\
\text { haciendo (AH), el } \\
\text { panamericanismo y un } \\
\text { fuerte sistema disciplinario. }\end{array}$ & $\begin{array}{l}\text { Programa de tres } \\
\text { años. Pocos siguieron } \\
\text { carreras } \\
\text { universitarias. }\end{array}$ & $\begin{array}{l}\text { AH como su } \\
\text { principal } \\
\text { enfoque, } \\
\text { diferenciándol } \\
\text { o de otras } \\
\text { instituciones } \\
\text { educativas. }\end{array}$ & No desarrollada & $\begin{array}{l}\text { Hay } 180 \text { estudian- } \\
\text { tes bajo un fuerte } \\
\text { sistema } \\
\text { disciplinario }\end{array}$ \\
\hline $\begin{array}{l}1958- \\
1978\end{array}$ & $\begin{array}{l}\text { Deja de ser una escuela } \\
\text { secundaria a ser una } \\
\text { escuela técnica. Inicio de } \\
\text { admisión basado en un } \\
\text { examen de admisión y } \\
\text { requerimiento del diploma } \\
\text { de secundaria. Estimula a } \\
\text { los graduados a seguir } \\
\text { estudiando para la } \\
\text { ingeniería y posgrados. }\end{array}$ & $\begin{array}{l}\text { Fortalecimiento de la } \\
\text { educación en las } \\
\text { ciencias. Creación del } \\
\text { Departamento de } \\
\text { Ciencias (1969) }\end{array}$ & $\begin{array}{l}\text { Establecimien- } \\
\text { to de un } \\
\text { programa de } \\
\text { trabajo } \\
\text { sistematizado. } \\
\text { Pioneros en la } \\
\text { inseminación } \\
\text { artificial en } \\
\text { bovinos } \\
\text { (1964) }\end{array}$ & $\begin{array}{l}\text { Capacitación a } \\
\text { productores en } \\
\text { temas agrícolas. } \\
\text { Creación de la } \\
\text { oficina } \\
\text { Desarrollo: } \\
\text { Relaciones } \\
\text { públicas } \\
\text { graduados (1968) }\end{array}$ & $\begin{array}{lr}\text { Calendario acadé- } \\
\text { mico pasa de } 9 \text { a } \\
11 \text { meses. Poco } \\
\text { aumento } & \text { en } \\
\text { número } & \text { de } \\
\text { estudiantes. } & \\
\text { Creación de } & \text { la } \\
\text { oficina } & \text { de } \\
\text { Zamorano } & \text { en } \\
\text { Boston (1969) } & \end{array}$ \\
\hline $\begin{array}{l}1979- \\
1987\end{array}$ & $\begin{array}{l}\text { Un graduado Zamorano } \\
\text { como nuevo director de la } \\
\text { EAP. Los cambios } \\
\text { generados por la nueva } \\
\text { dirección permiten en } 1981 \\
\text { el ingreso de mujeres a } \\
\text { Zamorano, Desarrollo del } \\
\text { Plan estratégico (1980) }\end{array}$ & $\begin{array}{l}\text { 4to año agregado } \\
\text { como opcional; Una } \\
\text { Maestría conjunta } \\
\text { con } \quad \text { Cornell } \\
\text { University para un } \\
\text { programa de estudios } \\
\text { profesionales } \\
\text { (finalizó en 1987) }\end{array}$ & $\begin{array}{l}\text { Se mantiene el } \\
\text { mismo sistema } \\
\text { de trabajo. }\end{array}$ & $\begin{array}{l}\text { Creación } \\
\text { programas } \\
\text { extensión. }\end{array}$ & $\begin{array}{l}\text { Incremento en el } \\
\text { número } \\
\text { estudiantes; } \\
\text { admisión } \\
\text { estudiantes } \\
\text { mujeres; } \\
\text { crecimiento de la } \\
\text { infraestructura. } \\
\end{array}$ \\
\hline $\begin{array}{l}1988- \\
1992\end{array}$ & $\begin{array}{l}\text { Crecimiento y consolida- } \\
\text { ción de los programas } \\
\text { académicos (6 departamen- } \\
\text { tos), motivando a los } \\
\text { graduados a buscar } \\
\text { maestrías principalmente } \\
\text { en áreas técnicas. Inicio de } \\
\text { las tesis de grado. }\end{array}$ & $\begin{array}{l}\text { Curriculum } \\
\text { reformado, cambio a } \\
\text { un sistema de } 3+1 \\
\text { para una opción de } \\
\text { ingeniería } \\
\text { agronómica (a nivel } \\
\text { de licenciatura) }\end{array}$ & $\begin{array}{l}\text { Inicio en } \\
\text { trabajos de } \\
\text { desarrollo } \\
\text { rural; módulos } \\
\text { administrati- } \\
\text { vos agregados } \\
\text { e incremento } \\
\text { de la fragmen- } \\
\text { tación de } \\
\text { módulos. } \\
\end{array}$ & $\begin{array}{l}\text { Proyecto } \\
\text { poscosecha; } \\
\text { Manejo integrado } \\
\text { de plagas en } \\
\text { Honduras } \\
\text { (MIPH); Proyecto } \\
\text { de Desarrollo } \\
\text { Rural }\end{array}$ & $\begin{array}{l}\text { Énfasis en la } \\
\text { expansión de las } \\
\text { instalaciones } \\
\text { físicas }\end{array}$ \\
\hline $\begin{array}{l}1993- \\
1995\end{array}$ & $\begin{array}{lr}\text { Iidentifican } & \text { oportunidades } \\
\text { de mejora, más acciones } \\
\text { orientadas al cliente } \\
\text { (estudiantes } \\
\text { empleadores). Preparación } \\
\text { del plan estratégico. } \\
\text { Aumenta el número de } \\
\text { aspirantes. }\end{array}$ & $\begin{array}{l}\text { Curriculum reforma- } \\
\text { do basado en el perfil } \\
\text { del graduado; Porta- } \\
\text { folio del docente } \\
\text { como herramienta de } \\
\text { evaluación y auto- } \\
\text { análisis. Estímulo al } \\
\text { aprendizaje activo e } \\
\text { interdisciplinaridad. }\end{array}$ & $\begin{array}{l}\text { Predominio de } \\
\text { micromódulos }\end{array}$ & $\begin{array}{l}\text { MIP en América } \\
\text { Central; inicio del } \\
\text { Proyecto } \\
\text { Desarrollo } \\
\text { Sostenible } \\
\text { Yeguare. }\end{array}$ & $\begin{array}{l}\text { El } 11 \% \text { de los } \\
\text { estudiantes son } \\
\text { mujeres; énfasis } \\
\text { en renovación de } \\
\text { instalaciones } \\
\text { físicas. }\end{array}$ \\
\hline
\end{tabular}


Cuadro 1. Continuación

\begin{tabular}{|c|c|c|c|c|c|}
\hline Período & Resumen & $\begin{array}{c}\text { Currículum } \\
\text { Formal }\end{array}$ & $\begin{array}{l}\text { Aprender } \\
\text { Haciendo }\end{array}$ & Proyección & $\begin{array}{c}\text { Ambiente del } \\
\text { Campus }\end{array}$ \\
\hline $\begin{array}{l}1996- \\
1997\end{array}$ & $\begin{array}{l}\text { Generación de un proceso } \\
\text { de introspección. Se recibe } \\
\text { a una comisión evaluadora } \\
\text { externa que apoya la } \\
\text { revisión. Desarrollo de } \\
\text { estudios de mercado y } \\
\text { análisis de las necesidades } \\
\text { de los empleadores }\end{array}$ & $\begin{array}{l}\text { Crecimiento de la } \\
\text { importancia } \\
\text { aprendizaje activo. }\end{array}$ & $\begin{array}{l}\text { Se crean los } \\
\text { macromó- } \\
\text { dulos. }\end{array}$ & $\begin{array}{l}\text { Inicio del apoyo a } \\
\text { microempresarios; y } \\
\text { establecimiento de } \\
\text { la Decanatura de } \\
\text { Proyección }\end{array}$ & $\begin{array}{l}\text { Creación de la } \\
\text { primera residencia } \\
\text { estudiantil con } \\
\text { padrinos. }\end{array}$ \\
\hline $\begin{array}{l}1998- \\
2001\end{array}$ & $\begin{array}{l}\text { Desarrollo y consolidación } \\
\text { de un perfil de graduado } \\
\text { basado en las necesidades } \\
\text { de la sociedad; Programa } 4 \\
\times 4 \text { aprobado. Esfuerzo } \\
\text { para consolidar el nuevo } \\
\text { sistema del } 4 \quad \times \quad 4 . \\
\text { Compromiso para mejorar } \\
\text { las metodologías de } \\
\text { enseñanza y modernizar las } \\
\text { expectativas sobre los } \\
\text { resultados del aprendizaje. }\end{array}$ & $\begin{array}{l}\text { Período de transición: } \\
\text { sigue el } 3+1 \text { para } \\
\text { graduar a estudiantes } \\
\text { en transición y } 4 \text { años } \\
\text { de duración del inicio } \\
\text { del Programa } 4 \times 4 \text {. } \\
\text { Fortalecimiento de } \\
\text { nuevas metodologías: } \\
\text { aprendizaje en grupo, } \\
\text { aprendizaje activo y } \\
\text { centrado en el } \\
\text { estudiante en el salón } \\
\text { de clases y el } \\
\text { Aprender Haciendo }\end{array}$ & $\begin{array}{l}\text { Creación de } \\
\text { Zamo- } \\
\text { empresas }\end{array}$ & $\begin{array}{l}\text { Programas de } \\
\text { recuperación Pos- } \\
\text { Huracán Mitch en } \\
\text { Honduras y Nicara- } \\
\text { gua; cuencas hidro- } \\
\text { gráficas y Agro- } \\
\text { negocios. Fortaleci- } \\
\text { miento de proyectos } \\
\text { con base educativa } \\
\text { en Centro y Sur } \\
\text { América. Se } \\
\text { elimina la Decana- } \\
\text { tura de Proyección; } \\
\text { establecimiento de } \\
\text { PROEMPREZAH }\end{array}$ & $\begin{array}{l}\text { Incremento de las } \\
\text { actividades los } \\
\text { extracurriculares. } \\
\text { Todos estudiantes viven } \\
\text { en residencias; } \\
\text { Inicia el Sistema } \\
\text { Integrado de Vida } \\
\text { Estudiantil } \\
\text { (SIVE) con guías. }\end{array}$ \\
\hline $\begin{array}{l}2002- \\
2010\end{array}$ & $\begin{array}{l}\text { Consolidado el programa } 4 \\
\times 4 \text {. Plan estratégico } 2006- \\
2010 \text { y crecimiento físico } \\
\text { del campus. }\end{array}$ & $\begin{array}{l}\text { Primera graduación } \\
\text { del } 4 \times 4 \text {. Currículo } \\
\text { reformado y } \\
\text { aprobado en el } 2005\end{array}$ & $\begin{array}{l}\text { Primeras } \\
\text { prácticas } \\
\text { fuera del } \\
\text { campus de } \\
\text { Zamorano. }\end{array}$ & $\begin{array}{ll}\text { Proyectos } & \text { de } \\
\text { Cuencas } & \\
\text { Hidrográficas } & \end{array}$ & \begin{tabular}{l}
\multicolumn{3}{l}{ Establecimiento } \\
de la Decanatura \\
Asociada de \\
Estudiantes y el \\
Programa de Vida \\
Estudiantil (PVE) \\
en lugar del \\
SIVE. \\
\end{tabular} \\
\hline $\begin{array}{l}2011- \\
2012\end{array}$ & $\begin{array}{l}\text { Nuevo Rector. Plan } \\
\text { Estratégico 2012-2016. }\end{array}$ & $\begin{array}{l}\text { Currículo reformado } \\
\text { y aprobado en el } \\
2011\end{array}$ & $\begin{array}{l}\text { Revisión del } \\
\text { Aprender } \\
\text { Haciendo } \\
\text { con enfoque } \\
\text { a calidad } \\
\text { educativa y } \\
\text { tecnología }\end{array}$ & & $\begin{array}{l}\text { Número más alto } \\
\text { de estudiantes } \\
\text { registrados en } \\
\text { primer ingreso, } \\
395 .\end{array}$ \\
\hline
\end{tabular}

Fuente: Adaptado por los autores (Andrews 2002, Malo 1999).

Para Zamorano, la vida estudiantil extracurricular es el currículo invisible que es un espacio de aprendizaje para la vida porque crea escenarios para desarrollar liderazgo y creatividad; al mismo tiempo, que los hace protagonistas de eventos y actividades que los enfrenta a desafíos que los forman integralmente. La institución pone mucha atención en la vida estudiantil y posee profesionales especializado para incentivar, promover valores y crear un ambiente propicio y agradable para el aprendizaje.

La institución también contribuye al desarrollo científico de la región en los programas de 
investigación y en proveer a los países especialistas que aportan a la solución de problemas del desarrollo agropecuario y agroindustrial, teniendo como resultado productos y procesos innovadores que han sido como punta de lanza para el mejoramiento de la agricultura tropical y que ha servido como material para la construcción de nuevo conocimiento interinstitucional y estudiantil.

El aporte en proyección es enorme, no sólo nacional, sino internacional, con una serie de programas y proyectos regionales que han apoyado a los países y han contribuido al combate de la pobreza y al detenimiento de la degradación ambiental. Todos los aspectos anteriores dan el escenario ideal para contribuir al mundo con graduados formados integralmente, con valores y pertinentes a las necesidades globales.

Zamorano ha servido de modelo para el desarrollo de instituciones educativas latinoamericanas. Tal como lo menciona el Dr. Malo en su libro El Zamorano-Afrontando el reto de la América Tropical (Malo, 1999): “imitar el modelo... ¡El mejor cumplido!". Esta es la mejor muestra de la contribución significativa que ha tenido Zamorano en sus 70 años. Algunos ejemplos son:

1. La Universidad Nacional de Agricultura (antes ENA), ubicada en Catacamas, Olancho, Honduras. Su Primer Director fue Gustavo Pérez Osorio, graduado Zamorano y discípulo del Dr. Wilson Popenoe. Esta universidad fue fundada 10 años después de Zamorano por insistencia del Presidente Juan Manuel Gálvez (Malo 1999, p. 352)

2. La Escuela Nacional de Agricultura de El Salvador, fundada en 1958, ofreció al Dr. W. Popenoe la dirección de la misma. Él no aceptó aduciendo que se lo impedía su edad. Algunos consideraron que la situación política del país también influyó (Malo 1999, p. 303)

3. En la primera década del presente siglo, Zamorano creo, a solicitud de la Fundación Juan Castello, la Unidad Educativa Fiscal del Milenium: Colegio Experimental Agropecuario Ing. Agr. Juan José Castelló Zambrano (en nombre del fallecido graduado Zamorano del mismo nombre (1986-2005), el bachillerato con opción de internado, en Las Juntas, Limoncito, Ecuador. Esta iniciativa ha tomado a la institución como un modelo académico y de gestión (Fundación Castelló, 2012).

El aporte del modelo Zamorano sigue siendo un referente a nivel mundial de educación agrícola y sin duda un modelo de pedagogía efectivo y pertinente.

\section{Los Graduados, Pieza Fundamental para la Contribución de Zamorano al Mundo}

Los más de 6,000 graduados de Zamorano provenientes de diferentes países (Cuadro 2), son el producto principal de Zamorano que por su empeño, preparación, disciplina, liderazgo y formación integral, han logrado establecer un prestigio internacional al convertirse en dueños de fincas y agroindustrias, directores y ejecutivos de corporaciones y bancos, académicos e investigadores, líderes de proyectos y organizaciones no gubernamentales de desarrollo, ministros de gobierno y otros profesionales también distinguidos y reconocidos por sus contribuciones al desarrollo de la sociedad. Más de 50 graduados se han desempeñado en altos cargos de gobiernos de la región como ministros o viceministros.

Nuestros graduados superan su desempeño académico en otras universidades, demostrando la alta calidad de la formación integral zamorana, ganando una excelente reputación en escuelas de posgrado de universidades de Estados Unidos, Centro América, Sur América, Europa y Asia. Los zamoranos son reconocidos por su mística, dedicación, versatilidad, perseverancia, productividad, voluntad de servicio y su constante superación personal y profesional. Los lazos fraternos desarrollados durante los estudios en Zamorano hacen que sus graduados sean muy unidos y conserven la amistad de por vida.

Los graduados están organizados en la Asociación de Graduados de la Escuela Agrícola Panamericana con capítulos y subcapítulos nacionales; ellos, además de dedicarse a sus actividades profesionales y familiares, aportan su tiempo, ideas y esfuerzo para contribuir en la difusión de este modelo educativo que tiene todos los componentes para seguir adelante y contribuir con el desarrollo del mundo. 
Cuadro 2. Distribución de graduados zamorano por país, 1946-2011.

\begin{tabular}{|c|c|c|c|c|c|}
\hline$\#$ & País & Graduados & $\#$ & País & Graduados \\
\hline 1 & Alemania & 1 & 16 & Guatemala & 688 \\
\hline 2 & Argentina & 2 & 17 & Haití & 18 \\
\hline 3 & Austria & 1 & 18 & Honduras & 1,820 \\
\hline 4 & Bélgica & 1 & 19 & Israel & 1 \\
\hline 5 & Belice & 70 & 20 & Italia & 3 \\
\hline 6 & Bolivia & 321 & 21 & Jamaica & 1 \\
\hline 7 & Brasil & 1 & 22 & México & 48 \\
\hline 8 & Chile & 14 & 23 & Nicaragua & 489 \\
\hline 9 & Colombia & 330 & 24 & Panamá & 257 \\
\hline 10 & Costa Rica & 410 & 25 & Paraguay & 15 \\
\hline 11 & Cuba & 20 & 26 & Perú & 84 \\
\hline 12 & Ecuador & 1,491 & 27 & República Dominicana & 145 \\
\hline 13 & El Salvador & 429 & 28 & Uruguay & 1 \\
\hline 14 & España & 3 & 29 & Venezuela & 29 \\
\hline 15 & Estados Unidos & 11 & & Total & 6,704 \\
\hline
\end{tabular}

\section{Contribuciones de Zamorano a América Latina}

Las acciones educativas, internas y externas, desarrolladas por Zamorano están fundamentadas no sólo en los conocimientos técnicos que manejan sus docentes, sino en las investigaciones y acciones en que se involucran, ya sean acompañadas por sus estudiantes o con beneficiarios externos con los que trabajan. Estos conocimientos son aplicados en las clases, investigación y proyección generando beneficio para los estudiantes y capacitados.

Zamorano exige, como parte de las actividades regulares de sus docentes, la participación en investigación y proyección. Ellos investigan y recaban nueva información y aplican técnicas innovadoras, mostrando al estudiante la aplicación de esta información en la vida real. Es aquí donde Zamorano va más allá de las condiciones controladas del ambiente educativo; los variantes escenarios de aplicación fuera de Zamorano permiten a los involucrados comparar y adaptar los conocimientos y técnicas a una realidad cambiante donde hay muchos involucrados, y donde se debe considerar la influencia de cada actividad en la zona donde se interviene.
Algunas de las áreas con las que Zamorano contribuye a las sociedades incluyen proyectos de investigación y proyección en temas como:

\section{Manejo Integrado de Plagas}

- Control Biológico. Reducción del impacto del uso de plaguicidas en los recursos naturales a través de la transformación de la agricultura tradicional utilizando productos y organismos naturales como hongos benéficos, enemigos naturales, nematodos entomopatógenos $\mathrm{y}$ ácaros depredadores.

- El Programa de Manejo Integrado de Plagas (PROMIPAC) fue una iniciativa del Departamento de Protección Vegetal a principios de los 90; logrando el auspicio de la Cooperación Suiza para el Desarrollo; se implementó un proyecto, que luego se convirtió en programa cubriendo Honduras, Nicaragua y El Salvador. Este Programa usó metodologías participativas con la visión de mejorar y reducir el uso de plaguicidas. Además, hacer más eficientes diversas prácticas agronómicas para reducir plagas y 
aumentar productividad. Ha sido un Programa conocido por su trabajo con pequeños $\mathrm{y}$ medianos productores con la metodología Escuelas de Campo (ECA) e Investigación Acción Participativa (IAP).

\section{Ambiente y Desarrollo}

- Cuencas Hidrográficas. Desde el nacimiento del departamento de Recursos Naturales en 1993, Zamorano ha trabajado en programas de investigación y proyección en cuencas hidrográficas con el auspicio de patrocinadores centrados en el desarrollo sostenible en el tema de calidad de agua y la sostenibilidad ambiental.

- Sostenibilidad ambiental. Desarrollo de proyectos para una gestión ambiental limpia, mejor aprovechamiento de los recursos naturales y las materias primas, reducción de residuos sólidos y líquidos.

- Energía renovable. Búsqueda de alternativas para la energía proveniente de combustibles fósiles y apropiada a las condiciones de las comunidades (investigación en energía solar, estufas mejoradas, biodigestores y otras energías alternativas).

- Biocombustibles. Investigación de oportunidades económicas para la producción de biocombustibles a partir de la Jatropha y otras biomasas.

- Biodiversidad. Se crea el Centro Zamorano de Biodiversidad (CZB), miembro de la IRBIO (Instituto Regional de Biodiversidad-SICA), para monitorear y evaluar la biodiversidad en Centroamérica, a la vez, que se impulsan iniciativas regionales para la protección de los ecosistemas y las especies.

- Desde la década de los ochentas, Zamorano inició su contribución en desarrollo a través del Programa de Desarrollo Rural (con cuatro sedes en comunidades aledañas); Proyecto UNIR (Auspiciado por la Fundación Kellogg para beneficiar al Valle del Yeguare) y el proyecto de Desarrollo Sostenible del Yeguare (incluyendo 11 municipios de la mancomunidad del Yeguare).
- Con la experiencia educativa agrícola se ha fortalecido la educación técnica a través de diagnósticos situacionales, el desarrollo de mallas curriculares, de manuales contextualizados, capacitación técnica y docente en el nivel medio de institutos públicos en Centro América y algunos de Sur América.

\section{Mejoramiento Genético de Plantas}

- Producción de semilla mejorada. Fortalece la producción de semilla mejorada en Centro América y contribuye con el Gobierno de Honduras al proveerle semilla destinada a productores del país.

- Programa de Investigación de Frijol (PIF). Por más de 20 años se ha investigado y liberado nuevas variedades de frijol con mejores características nutricionales, resistentes a la sequía, así como producción de germoplasma mejorado para distribución a la Red de Frijol en Centroamérica y el Caribe, contribuyendo a reducir los niveles de inseguridad alimentaria de los países de la región.

\section{Producción Animal}

- Producción de animales. Los docentes han desarrollado material genético animal que ha servido como referente centroamericano para el mejoramiento genético de fincas y granjas.

- Ganado lechero. Desde su nacimiento, Zamorano ha desarrollado la unidad de ganado lechero. En su trayectoria, esta unidad ha servido como referente internacional en hatos altamente productivos, contando con más de 40 años de experiencia en inseminación artificial, en las razas más productivas en el mundo (Jersey, Holstein y Pardo suizo). Un sinnúmero de productores de diferentes países han sido capacitados por esta unidad.

- Ganado porcino. Desde los primeros años de su fundación, Zamorano ha aportado a los países de Centro América cerdos reproductores de excelente calidad, y este aporte se ha incrementado a partir del año 2000 con la implementación de un programa 
de mejora genética importando semen desde Norte América.

- Desde el 2006, Zamorano le ha apostado al desarrollo de la avicultura latinoamericana a través del Centro de Investigación y Enseñanza Avícola de Zamorano. En este centro, los estudiantes además de aprender de producción de huevos y carne de pollo, se exponen a investigación científica de alto nivel; ya que el centro desarrolla investigaciones para empresas productoras avícolas, empresas productoras de alimentos y micronutrientes, universidades y otros centros de investigación. Adicionalmente, es una unidad donde convergen productores $\mathrm{e}$ investigadores para construir nuevo conocimiento avícola.

\section{Fortalecimiento de las PYMES}

- PROEMPREZAH. Este proyecto abarcó ocho departamentos de Honduras y contribuyó a la transformación de pequeños y medianos productores agrícolas y micro y pequeños empresarios agroindustriales del sector rural y semiurbano para convertirlos en empresarios exitosos y responsables.

- Incremento de la competitividad de las cadenas de valor a través del fortalecimiento de PYMES agroindustriales a nivel centroamericano. Fortaleciendo la capacidad de los productores, procesadores y comercializadores para mejorar su acceso a mercados, incrementar su productividad y mejorar su capacidad de gestión.

En adición a lo anterior, y como parte de su contribución a la sociedad, Zamorano publica sus hallazgos, algunas de éstas se hacen en conjunto con otras organizaciones con las que se colabora, otras son el resultado de trabajos de Proyectos de graduación (tesis) escritas por los estudiantes que a menudo producen nuevas tecnologías para resolver problemas de su área de especialización. Además, existen las publicaciones técnicas en la revista Ceiba que pertenece a Zamorano; y las que publican los docentes, como ejemplo los centenares de artículos de investigación publicados por los docentes en revistas científicas en áreas como agronomía, ganadería, agroindustria, mejoramiento genético y biotecnología.

Resaltan también las publicaciones bibliográficas de los docentes que sirven de guía y referencia a nivel latinoamericano, algunas son Producción de Ganado Lechero en el Trópico (Vélez et al., 2009), Tecnología de la Leche (Revilla, 2009.), Producción de Cerdos (Castillo, 2006), Introducción a la Acuacultura (Meyer, 2003), entre otros.

\section{Literatura Citada}

Andrews, K. 2004. The deep change process in Zamorano: 1997-2002. A case study. UNESCO, International Institute for Educational Planning. Paris, France. Recuperado el 23 de marzo, 2012 de http://unesdoc.unesco.org/images/0013/001360/136069 e.pdf

Castillo, R. 2006. Producción de Cerdos. Zamorano Academic Press, Zamorano, Honduras. 89 p.

Fundación Castelló. Consultado el 20 de mayo de 2012. Disponible en http://www.juanjose06.org/

Malo, S.E. 1999. El Zamorano-Afrontando el reto de la América Tropical. 1999. Manhattan, Kansas, Estados Unidos. Simbad Books.

Meyer, D. 2003. "Construcción de estanques". Manual Técnico. Escuela Agrícola Panamericana, Zamorano, Honduras.

http://www.acuacultura.org/production/estanques_mas_ info.htm

Revilla, A. 2009. Tecnología de la leche. Zamorano Academic Press, Zamorano, Honduras. 312 p.

Vélez, M., Hincapié, J.J., Matamoros, I. 2009. Producción de Ganado Lechero en el Trópico. 6ta. edición. Zamorano Academic Press, Zamorano, Honduras. 294 p.

Vélez, M. 1993. Producción de Cabras y Ovejas en el Trópico. Zamorano, Honduras. 174 p.

Recibido para publicación el 20 de abril de 2012. Aceptado para publicación el 21 de enero de 2013. 